\title{
Downregulated MEG3 participates in rheumatoid arthritis via promoting proliferation of fibroblast-like synoviocytes
}

\author{
XIN LU and JUN QIAN \\ Department of Orthopaedics, Peking Union Medical College Hospital, Chinese Academy of \\ Medical Sciences and Peking Union Medical College, Beijing 100730, P.R. China
}

Received May 25, 2018; Accepted December 5, 2018

DOI: $10.3892 /$ etm.2018.7100

\begin{abstract}
Maternally expressed gene 3 (MEG3) in rheumatoid arthritis (RA) and its underlying mechanism were explored. Synovial tissues from 10 RA patients and 10 controls were collected to detect MEG3 expression in fibroblast-like synoviocytes (FLS). The relationship between MEG3 expression and TNF- $\alpha$ was analyzed. After MEG3 knockdown by lentivirus transfection, cell cycle, proliferation, apoptosis, invasion and secretion of inflammatory factors were detected. Furthermore, the effect of MEG3 on STAT3 and PI3K/AKT pathways was explored. MEG3 was downregulated in RA patients, and exogenous TNF- $\alpha$ treatment could decrease MEG3 expression. After transfection with lentivirus, downregulated MEG3 led to FLS proliferation and secretion of inflammatory cytokines, IL-6 and IL-8, improved the invasive ability and inhibited apoptosis. Reverse transcription-quantitative polymerase chain reaction (RT-qPCR) results revealed that downregulated MEG3 increased the expression levels of MMP2 and MMP9. Western blotting results showed that downregulated MEG3 activated STAT3 and PI3K/AKT pathways. Downregulated MEG3 was able to promote proliferation and invasion, and inhibit apoptosis of FLS via STAT3 pathway.
\end{abstract}

\section{Introduction}

Rheumatoid arthritis (RA) is a common chronic and autoimmune disease, which is mainly manifested as the joint involvement, ultimately resulting in joint destruction, deformity and limb movement disorders. RA leads to a heavy economic and psychological burden on patients and society. Therefore, it is of great significance to improve the early diagnosis,

Correspondence to: Dr Jun Qian, Department of Orthopaedics, Peking Union Medical College Hospital, Chinese Academy of Medical Sciences and Peking Union Medical College, 1 Shuaifuyuan, Wangfujing, Dongcheng, Beijing 100730, P.R. China

E-mail:qj@medmail.com.cn

Key words: MEG3, proliferation, apoptosis, invasion, STAT3 timely treatment and effective control of RA progression (1). Recent studies have confirmed that the abnormal proliferation of T-lymphocytes and fibroblast-like synoviocytes (FLS) is the main cause of RA $(2,3)$. Therefore, inhibition of FLS may assist with improvement of the treatment efficacy of RA. In addition to the stimulation of inflammatory factors, FLS apoptosis is also an essential cause of abnormal proliferation of RA (4).

Long non-coding RNA (lncRNA) is a kind of RNA molecule with $>200$ nucleotides in length, which does not have the function of translating proteins (5). Initially, researches on non-coding RNAs were mainly focused on microRNAs, and IncRNAs were considered to be only waste products of the genome. However, accumulating studies have shown that lncRNAs are greatly involved in biological processes. Studies have found that IncRNA expression is tissue- and cell-specific, which is capable of regulating gene expression levels and cellular processes at epigenetic, transcriptional and post-transcriptional levels $(6,7)$.

Maternally expressed gene 3 (MEG3) is a human homologue of the mouse maternal gene Gt12, which is highly conserved in evolution. MEG3 is located on human chromosome $14 \mathrm{q} 32.3$ with $\sim 1.6 \mathrm{~kb}$ in length. Genome structural analysis has revealed that MEG3/Glt2 consists of 10 exons. Studies have confirmed that MEG3 is not a typical antisense sequence of gene transcript, but belongs to a kind of regulatory lncRNA with tumor suppressor function (8). MEG3 is found to be overexpressed in normal tissues such as the pituitary gland, brain tissue, and placenta. Downregulated or absent MEG3 is observed in tumors, such as liver and lung cancer. MEG3 is also associated with tumor grade and prognosis. Functionally, MEG3 participates in cell cycle, proliferation, metastasis and apoptosis of tumor cells $(9,10)$. The role of MEG3 in RA, however, needs to be further elucidated.

\section{Patients and methods}

Cell isolation and culture of FLS. Ten RA patients who received knee arthroscopic surgery and 10 trauma patients who received knee arthroscopic surgery in Peking Union Medical College Hospital (Beijing, China) were selected. The 10 RA patients were 3 males and 7 females, $51.1 \pm 12.0$ years of age. The 10 trauma patients were 8 males and 2 females, $41.4 \pm 12.7$ years of age. This study was approved by the Ethics 
Committee of Peking Union Medical College Hospital. Signed informed consents were obtained from the patients or the guardians.

Synovial tissues were collected during knee arthroscopic surgery. After grinding, digestion and centrifugation at $2,500 \mathrm{x} \mathrm{g}$ at $20^{\circ} \mathrm{C}$ for $10 \mathrm{~min}$, FLS were cultured in Dulbecco's modified Eagle's medium (DMEM) containing 10\% fetal bovine serum (FBS) (both from Gibco; Thermo Fisher Scientific, Inc., Waltham, MA, USA), $100 \mathrm{U} / \mathrm{ml}$ penicillin and $100 \mu \mathrm{g} / \mathrm{ml}$ streptomycin (HyClone; GE Healthcare, Chicago, IL, USA), and were incubated in a $5 \% \mathrm{CO}_{2}$ incubator at $37^{\circ} \mathrm{C}$. Cells in logarithmic growth phase were used for the following experiments after cell passage 4-8 times.

Cell transfection. Cells were transfected with LV-Vector or LV-shMEG3, after cell confluence was up to $60 \%$. Polybrene was added at a final dose of $4 \mu \mathrm{g} / \mathrm{ml}$. Fresh medium was replaced every 4-6 h. All lentiviruses were purchased from GenePharm (Shanghai, China).

$T N F-\alpha$ treatment. TNF- $\alpha(10 \mu \mathrm{g})$ was dissolved in sterile water to a final dose of $500 \mathrm{ng} / \mu 1$, which was then collected into $200 \mu \mathrm{l} \mathrm{EP}$ tubes and preserved at $-20^{\circ} \mathrm{C}$. Cells were treated with $10 \mathrm{ng} / \mathrm{ml}$ recombinant TNF- $\alpha$ for $24 \mathrm{~h}$, followed by determination of inflammatory factors and related genes in FLS.

Cell proliferation assay. Cell suspension was prepared and seeded into 96 -well plates with $4 \times 10^{3} /$ well. Cell Counting Kit-8 (CCK-8) solution (10 $\mu$ l) (Dojindo, Kumamoto, Japan) was added in each well after cells were cultured for 24,48 and $96 \mathrm{~h}$, respectively. The absorbance of each sample at $450 \mathrm{~nm}$ was measured by a microplate reader (Bio-Rad Laboratories, Inc., Hercules, CA, USA).

Cell apoptosis assay. For cell apoptosis detection, $200 \mu \mathrm{l}$ of binding buffer were added to prepare single cell suspension. In total $5 \mu \mathrm{l}$ of Annexin V-APC and $10 \mu \mathrm{l}$ of 7-AAD (Thermo Fisher Scientific, Inc.) were mixed in cell suspension and incubated in the dark for $15 \mathrm{~min}$, followed by cell apoptosis detection using flow cytometry (BD Biosciences, Franklin Lakes, NJ, USA). The data were analyzed using FlowJo software (FlowJo LLC, Ashland, OR, USA).

Reverse transcription-quantitative polymerase chain reaction $(R T-q P C R)$. Total RNA was extracted from cells by TRIzol reagent (Invitrogen; Thermo Fisher Scientific, Inc.) and then transcribed into complementary deoxyribonucleic acid (cDNA) using the PrimeScript RT reagent kit (Takara Biotechnology Co., Ltd., Dalian, China). qPCR was subsequently performed in strict accordance with the manufacturer's instructions of SYBR Green Real-Time PCR Master Mix (Invitrogen; Thermo Fisher Scientific, Inc.), with a total reaction volume of $10 \mu \mathrm{l}$. The following thermocycling conditions were used: denaturation at $95^{\circ} \mathrm{C}$ for $1 \mathrm{~min}$, annealing at $95^{\circ} \mathrm{C}$ for $30 \mathrm{sec}$ and extension at $60^{\circ} \mathrm{C}$ for $40 \mathrm{sec}$, for a total of 40 cycles. GAPDH served as the internal control. The relative expression levels of genes were expressed as $2^{-\Delta \Delta C q}(11)$. The primer sequences used in this study were as follows: MMP1 forward, AAAATTACACGCCAGATTGCC and reverse, GGTGTGACATTACTCCAGAGTTG; MMP2 forward, GAG
GAGCAGTTACGGTCTGTG and reverse, TCCTTTCCTTA GCTGACACTTGT; MMP3 forward, AGTCTTCCAATCCT ACTGTTGCT and reverse, TCCCCGTCACCTCCAATCC; MMP9 forward, TGTACCGCTATGGTTACACTCG and reverse, TGGCTTCCATAGAGTTCCTTCC; MEG3 forward, CAGCCAGAGTTAGCACAATAGG and reverse, CTGTTG TTCCCGTCGGAGTT; GAPDH forward, AGGTCGGTG TGAACGGATTTG and reverse, TGTAGACCATGTAGT TGAGGTCA.

Western blotting. Total protein was extracted from the treated cells by radioimmunoprecipitation assay (RIPA) solution (Roche Diagnostics, Basel, Switzerland). Total protein concentration was calculated by bicinchoninic acid (BCA) Protein Assay kit (Pierce; Thermo Fisher Scientific, Inc., Rockford, IL, USA). The protein sample was separated by electrophoresis on $10 \%$ sodium dodecyl sulphate-polyacrylamide gel electrophoresis (SDS-PAGE) and then transferred to a polyvinylidene fluoride (PVDF) membrane (EMD Millipore, Billerica, MA, USA). A total of $30 \mu \mathrm{g}$ of protein were added per lane for the electrophoresis. After the membranes were blocked with $5 \% \mathrm{BSA}$ at $20^{\circ} \mathrm{C}$ for $1 \mathrm{~h}$, they were incubated with primary antibodies overnight at $4^{\circ} \mathrm{C}$. The membranes were then washed with Tris-buffered saline with Tween-20 (TBST) and followed by incubation of secondary antibody. Immunoreactive bands were visualized by enhanced chemiluminescence (ECL) detection kit (Amersham; GE Healthcare, Foster City, CA, USA). The gray value was analyzed using ImageJ software (version 1.38; National Institutes of Health, Bethesda, MD, USA). Primary mouse monoclonal STAT3 antibody (dilution, 1/500; cat. no. ab119352); rabbit polyclonal PI3K antibody (dilution, 1/500; cat. no. ab70912); rabbit polyclonal AKT antibody (dilution, 1:500; cat. no. ab8805); rabbit monoclonal Bax antibody (dilution, 1/500; cat. no. ab32503); rabbit polyclonal Bcl-2 antibody (dilution, 1/500; cat. no. ab59348); rabbit polyclonal GAPDH antibody (dilution, 1:500; cat. no. ab37168) and secondary goat anti-rabbit (HRP) IgG antibody (dilution, 1:2,000; cat. no. ab6721) were all purchased from Abcam (Cambridge, MA, USA).

Enzyme-linked immunosorbent assay (ELISA). FLS in good cell growth were collected and seeded into 6-well plates at a dose of $5 \times 10^{3} /$ well, with 2 replicates in each group. Cells were treated with TNF- $\alpha$ for $24 \mathrm{~h}$, followed by supernatant collection. Vascular endothelial growth factor (VEGF) expression in cell supernatant was detected according to the manufacturer's instructions of ELISA kit (BioLegend, Inc., San Diego, CA, USA). Each experiment was repeated 3 times.

Transwell assay. The upper Transwell chamber was previously coated with Matrigel (BD Biosciences, San Jose, CA, USA) and maintained in an incubator for $2 \mathrm{~h}$. A total of $20 \mu \mathrm{l}$ of cell supernatant and $500 \mu 1$ of DMEM containing 5\% FBS were then added in the upper and lower chamber, respectively. Transwell chamber was removed after $24 \mathrm{~h}$-incubation, and the non-migrated cells in the chamber were gently wiped off with a cotton swab. The chamber was fixed with methanol for $15 \mathrm{~min}$, washed with PBS twice and stained in $1 \%$ crystal violet for $30 \mathrm{~min}$. Finally, 5 randomly selected fields were 
A

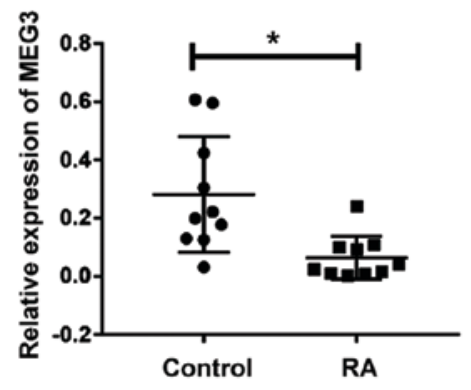

B

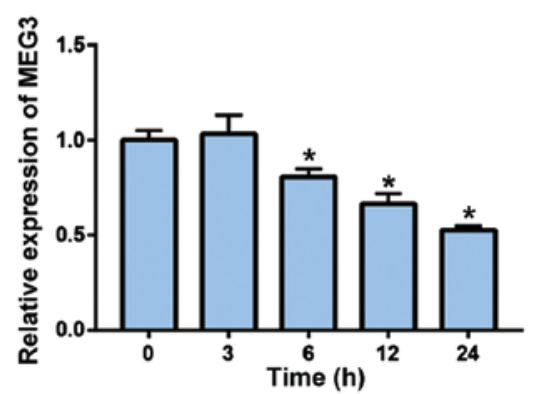

C

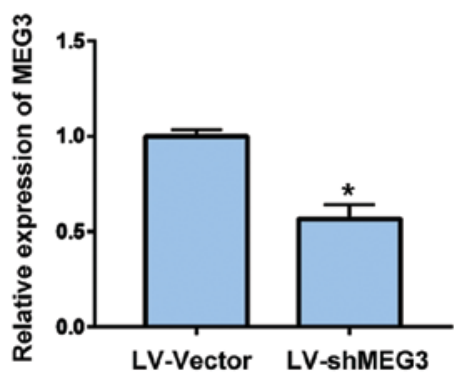

Figure 1. MEG3 expression levels in RA patients. (A) MEG3 expression levels in FLS of RA patients and controls. (B) Effect of TNF- $\alpha$ on MEG3 expression. (C) MEG3 expression after transfection of cells with LV-Vector or LV-shMEG3. * $\mathrm{P}<0.05$. MEG3, maternally expressed gene 3; RA, rheumatoid arthritis; FLS, fibroblast-like synoviocytes.

captured under an inverted microscope (magnification, $\mathrm{x} 40$; type, AZ100; Nikon Corp., Tokyo, Japan) for cell count.

Statistical analysis. All statistical analyses were conducted using Statistical Product and Service Solutions (SPSS) 16.0 software (SPSS, Inc., Chicago, IL, USA). Measurement data were expressed as mean \pm standard deviation. Independent-sample t-test was used to compare the differences between two groups. $\mathrm{P}<0.05$ was considered to indicate a statistically significant difference.

\section{Results}

MEG3 is downregulated in FLS of RA patients. We first extracted mRNAs of FLS from RA patients and controls. The results indicated that the mRNA level of MEG3 was downregulated in FLS of RA patients compared with that of controls (Fig. 1A). In vitro experiments also showed that TNF- $\alpha$ treatment could remarkably inhibit MEG3 expression in FLS in a time-dependent manner, which achieved the lowest level at $24 \mathrm{~h}$ (Fig. 1B). We therefore utilized lentivirus transfection to decrease MEG3 expression in FLS, so as to further explore the underlying potential of MEG3 (Fig. 1C).

Downregulated MEG3 promotes proliferation and invasion of FLS. The proliferative ability of FLS was remarkably increased after MEG3 knockdown, which achieved the peak at $72 \mathrm{~h}$ (Fig. 2A). Cell apoptosis results demonstrated a decreased apoptotic rate in FLS after MEG3 was inhibited (Fig. 2B). No significant difference was observed in cell cycle after MEG3 knockdown (Fig. 2C). Furthermore, the Transwell assay elucidated that downregulated MEG3 could remarkably promote the invasive ability of FLS compared to that of negative (Fig. 2D). Previous studies have pointed out that matrix metalloproteinases (MMPs) are involved in the development and progression of RA. Hence, we speculated that MEG3 could affect MMPs expression levels. Our data demonstrated that downregulated MEG3 could lead to increased expression levels of MMP2 and MMP9, but there were no significant differences in MMP1 and MMP3 (Fig. 2E). Cytokine secretion was also detected and the results revealed that downregulated MEG3 could remarkably promote the secretion of IL-6 (Fig. 2F) and IL-8 (Fig. 2G) after TNF- $\alpha$ treatment.
Downregulated MEG3 stimulates STAT3 pathway. It has been reported that STAT3 is greatly involved in cell apoptosis of RA (12). As a consequence, we speculated that MEG3 could regulate RA via STAT3 pathway. In the present study, our data indicated that downregulated MEG3 could not only promote the phosphorylation of STAT3 (Fig. 3A), but also regulate the expression levels of the downstream factors of STAT3 (Fig. 3B). Considering the crucial role of PI3K/AKT in cell proliferation, we detected the key factors in this pathway and found that downregulated MEG3 was also capable of stimulating the PI3K/AKT pathway (Fig. 3C).

\section{Discussion}

RA is a systemic autoimmune disease, which is characterized by synovial hyperplasia, articular cartilage destruction and subchondral bone erosion (13). Interaction of various immune cells and inflammatory factors leads to FLS activation when they are under various stimuli, such as the environment change, smoking and sex hormones alteration. Activated FLS in turn secrete a variety of chemokines and inflammatory factors, which further stimulate FLS proliferation from the original 4 layers to 10 layers. The continuously proliferating and activating FLS gradually transform into RA-FLS, which present the characteristics of tumor cells such as reduced apoptosis, enhanced invasiveness, and secretion of cytokines, MMPs and proteoglyases, thereby destroying structures of articular cartilage and bone (14). In this experiment, we found that MEG3 was downregulated in FLS of RA patients. In vitro experiments showed that TNF- $\alpha$ negatively regulates MEG3 expression. Also, downregulated MEG3 was found to lead to FLS proliferation, secretion of IL-6 and IL-8, increased expression levels of MMP2 and MM9, and to inhibite FLS apoptosis.

Our results also showed that downregulated MEG3 activates STAT3 pathway. It has been reported that STAT3 possesses two phosphorylation sites that are related to cellular function, namely STAT3 (Y705) and STAT3 (S727) (15). STAT3 is presented in the cytoplasm in an inactive form. Once the tyrosine residue of STAT3 (Tyr705) is phosphorylated, STAT3 induces the phosphorylation of tyrosine-SH2 region to form a STAT3 dimer. The STAT3 dimer further translocates into the nucleus and binds to STAT-specific DNA reaction fragments, thus activating a series of downstream 


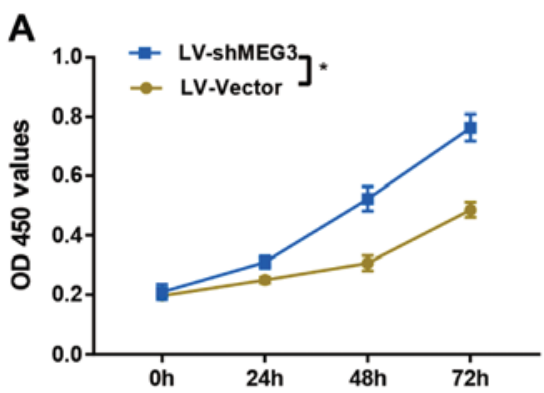

D

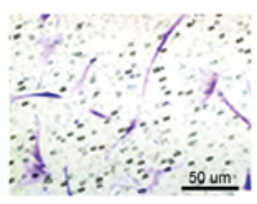

IV-Vector

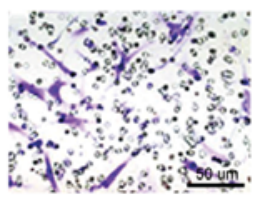

LV-shMEG3

F

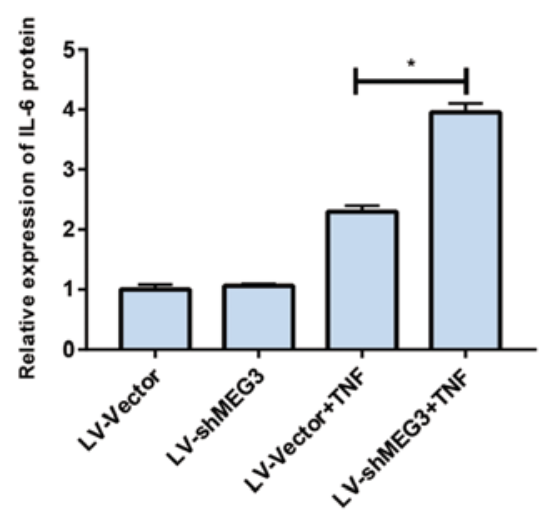

B
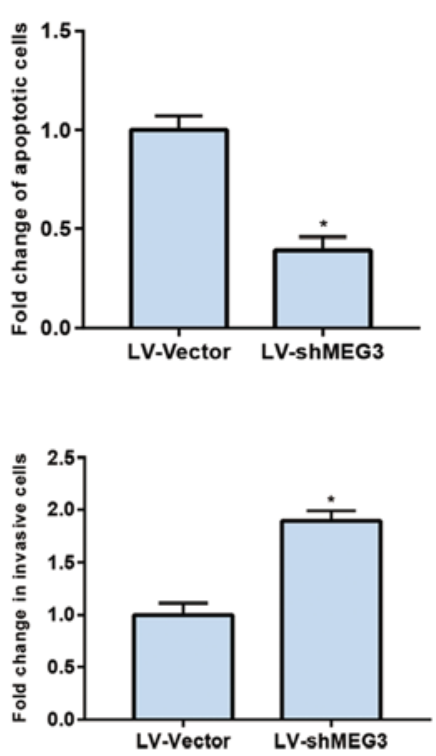

G

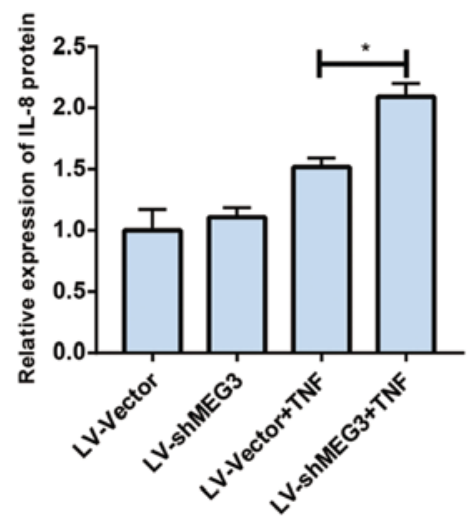

C

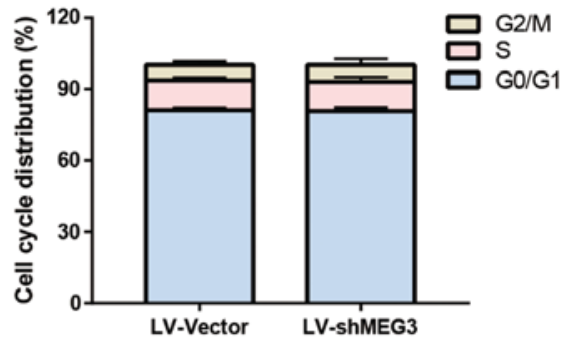

E

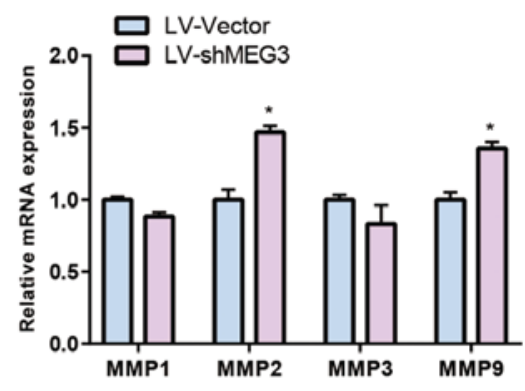

Figure 2. Downregulated MEG3 promotes proliferation and invasion of FLS. (A) Cell proliferation, (B) apoptosis, (C) cell cycle, and (D) invasion after downregulation of MEG3 (scale bar, $50 \mu \mathrm{m}$ ). (E) Expression levels of MMPs after downregulation of MEG3. Secretion of (F) IL-6 and (G) IL-8 after downregulation of MEG3. "P<0.05. MEG3, maternally expressed gene 3; FLS, fibroblast-like synoviocytes.

A

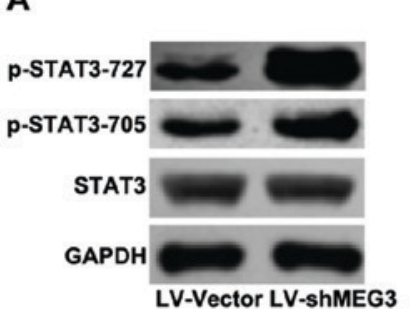

B

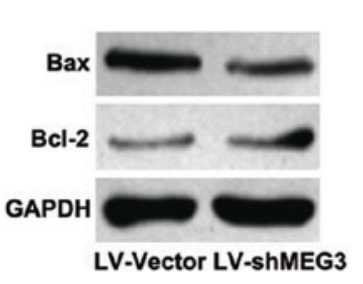

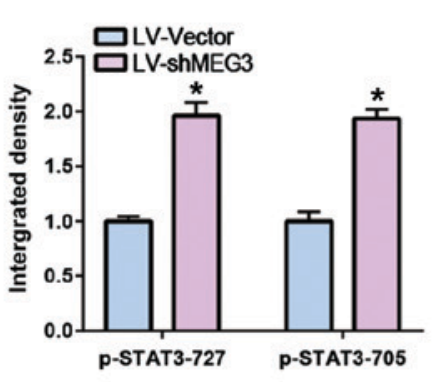

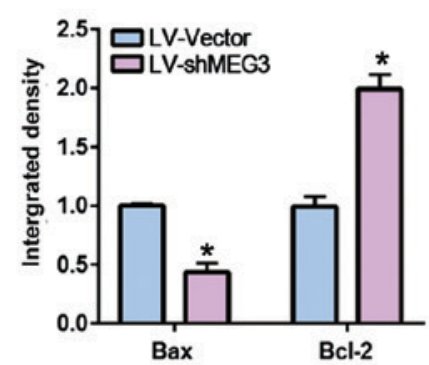

C
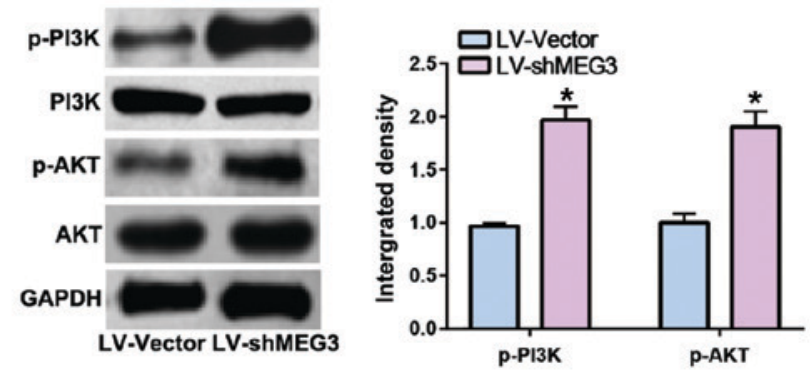

Figure 3. Downregulated MEG3 stimulates STAT3 pathway. Expression levels of (A) phosphorylated STAT3, and (B) Bcl-2 and Bax after downregulation of MEG3. (C) Effect of downregulated MEG3 on phosphorylation of PI3K/AKT pathway. ${ }^{*}<0.05$. MEG3, maternally expressed gene 3. 
gene expression levels $(16,17)$. Our experiment confirmed that MEG3 results in increased expression levels of RA-FLS, pSTAT3-705 and pSTAT3-727 in vitro. Moreover, downregulated MEG3 activates STAT3 pathway in RA-FLS.

$\mathrm{Bcl}-2$ is a member of the Bcl-2 family with anti-apoptotic capacity, which is an essential downstream of STAT3 pathway $(18,19)$. Herein, we found that $\mathrm{Bcl}-2$ is related to RA-FLS apoptosis. In vivo studies showed a higher Bcl-2 expression in the synovium of RA patients than that of osteoarthritis patients. Previous studies have confirmed that the stability of mitochondrial RA-FLS is regulated by Bcl-2, which exerts a crucial role in cell viability. Downregulated Bcl-2 can increase mitochondrial permeability and induce apoptosis (20). In vitro studies have shown that TNF and IL-1 can induce $\mathrm{Bcl}-2$ expression in RA-FLS, which protects RA-FLS in the inflammatory microenvironment (21). In this study, downregulated MEG3 led to an increased expression of Bcl-2 and decreased expression of Bax that promotes cell apoptosis of RA-FLS.

In addition to the excessive proliferation of FLS, degradation of articular cartilage is also one of the most serious pathological features of RA, which may be explained by the overactivation of proteolytic enzyme system. Among them, MMPs are widely expressed in RA-FLS. MMPs are a group of zinc-dependent endopeptidases that degrade various components of the extracellular matrix and are major proteases involved in cell migration and invasion $(22,23)$. Multiple members of the MMPs family have already been reported to be associated with RA, such as collagenase MMP1 and matrix lysin MMP3. The gelatinase subfamily includes two members, MMP2 and MMP9, which can digest collagenase-induced denatured collagen. MMP2 and MMP9 can also digest other matrix components, including fibrillar collagen I and II, and proteoglycans, thus participating in collagen degradation $(24,25)$. Studies have confirmed that MMP2 and MMP9 are remarkably upregulated in RA and are closely related to the erosion of articular cartilage (26). This experiment elucidated that downregulated MEG3 remarkably increases the expression levels of MMP2 and MMP9, but has no significant effect on MMP1 and MMP3.

In conclusion, downregulated MEG3 promotes proliferation and invasion, and inhibits apoptosis of FLS via STAT3 pathway.

\section{Acknowledgements}

Not applicable.

\section{Funding}

No funding was received.

\section{Availability of data and materials}

All data generated or analyzed during this study are included in this published article.

\section{Authors' contributions}

XL and JQ designed the study, performed the experiments, collected and analyzed the data. XL prepared the manuscript. All authors read and approved the final manuscript.

\section{Ethics approval and consent to participate}

This study was approved by the Ethics Committee of Peking Union Medical College Hospital (Beijing, China). Signed informed consents were obtained from the patients or the guardians.

\section{Patient consent for publication}

Not applicable.

\section{Competing interests}

The authors declare that they have no competing interests.

\section{References}

1. Di Spigna G, Del Puente A, Covelli B, Abete E, Varriale E, Salzano S and Postiglione L: Vitamin D receptor polymorphisms as tool for early screening of severe bone loss in women patients with rheumatoid arthritis. Eur Rev Med Pharmacol Sci 20: 4664-4669, 2016.

2. Calabrò A, Caterino AL, Elefante E, Valentini V, Vitale A, Talarico R, Cantarini L and Frediani B: One year in review 2016: Novelties in the treatment of rheumatoid arthritis. Clin Exp Rheumatol 34: 357-372, 2016.

3. Bellucci E, Terenzi R, La Paglia GM, Gentileschi S, Tripoli A, Tani C and Alunno A: One year in review 2016: Pathogenesis of rheumatoid arthritis. Clin Exp Rheumatol 34: 793-801, 2016.

4. Flurey CA, Hewlett S, Rodham K, White A, Noddings R and Kirwan J: Men, rheumatoid arthritis, psychosocial impact and self-management: A narrative review. J Health Psychol 21: 2168-2182, 2016

5. Wang C, Wang L, Ding Y, Lu X, Zhang G, Yang J, Zheng H, Wang $\mathrm{H}$, Jiang Y and Xu L: LncRNA structural characteristics in epigenetic regulation. Int J Mol Sci 18: 2659, 2017.

6. Peng WX, Koirala P and Mo YY: LncRNA-mediated regulation of cell signaling in cancer. Oncogene 36: 5661-5667, 2017.

7. Frank S, Aguirre A, Hescheler J and Kurian L: A lncRNA perspective into (re)building the heart. Front Cell Dev Biol 4: 128,2016

8. Zhou Y, Zhang X and Klibanski A: MEG3 noncoding RNA: A tumor suppressor. J Mol Endocrinol 48: R45-R53, 2012.

9. Balik V, Srovnal J, Sulla I, Kalita O, Foltanova T, Vaverka M, Hrabalek L and Hajduch M: MEG3: A novel long noncoding potentially tumour-suppressing RNA in meningiomas. J Neurooncol 112: 1-8, 2013.

10. Benetatos L, Voulgaris E and Vartholomatos G: DLK1-MEG3 imprinted domain microRNAs in cancer biology. Crit Rev Eukaryot Gene Expr 22: 1-15, 2012.

11. Livak KJ and Schmittgen TD: Analysis of relative gene expression data using real-time quantitative PCR and the 2(-Delta Delta C(T)) method. Methods 25: 402-408, 2001.

12. Krause A, Scaletta N, Ji JD and Ivashkiv LB: Rheumatoid arthritis synoviocyte survival is dependent on Stat3. J Immunol 169: 6610-6616, 2002.

13. Deane KD, Demoruelle MK, Kelmenson LB, Kuhn KA, Norris JM and Holers VM: Genetic and environmental risk factors for rheumatoid arthritis. Best Pract Res Clin Rheumatol 31: 3-18, 2017.

14. Fu H, Hu D, Zhang L and Tang P: Role of extracellular vesicles in rheumatoid arthritis. Mol Immunol 93: 125-132, 2018.

15. Sehgal PB: Paradigm shifts in the cell biology of STAT signaling. Semin Cell Dev Biol 19: 329-340, 2008.

16. Tebbutt NC, Giraud AS, Inglese M, Jenkins B, Waring P, Clay FJ, Malki S, Alderman BM, Grail D, Hollande F, et al: Reciprocal regulation of gastrointestinal homeostasis by SHP2 and STAT-mediated trefoil gene activation in gp130 mutant mice. Nat Med 8: 1089-1097, 2002.

17. Ranger JJ, Levy DE, Shahalizadeh S, Hallett M and Muller WJ: Identification of a Stat3-dependent transcription regulatory network involved in metastatic progression. Cancer Res 69: 6823-6830, 2009. 
18. Matsumoto S, Müller-Ladner U, Gay RE, Nishioka K and Gay S: Ultrastructural demonstration of apoptosis, Fas and Bcl-2 expression of rheumatoid synovial fibroblasts. J Rheumatol 23 : 1345-1352, 1996.

19. Yu H and Jove R: The STATs of cancer - new molecular targets come of age. Nat Rev Cancer 4: 97-105, 2004.

20. Perlman H, Georganas C, Pagliari LJ, Koch AE, Haines K III and Pope RM: Bcl-2 expression in synovial fibroblasts is essential for maintaining mitochondrial homeostasis and cell viability. J Immunol 164: 5227-5235, 2000.

21. Korb A, Pavenstädt H and Pap T: Cell death in rheumatoid arthritis. Apoptosis 14: 447-454, 2009.

22. Tolboom TC, van der Helm-Van Mil AH, Nelissen RG, Breedveld FC, Toes RE and Huizinga TW: Invasiveness of fibroblast-like synoviocytes is an individual patient characteristic associated with the rate of joint destruction in patients with rheumatoid arthritis. Arthritis Rheum 52: 1999-2002, 2005.
23. Ishiguro N: Cartilage degradation in rheumatoid arthritis. Clin Calcium 19: 347-354, 2009 (In Japanese).

24. Eck SM, Blackburn JS, Schmucker AC, Burrage PS and Brinckerhoff CE: Matrix metalloproteinase and G protein coupled receptors: Co-conspirators in the pathogenesis of autoimmune disease and cancer. J Autoimmun 33: 214-221, 2009.

25. Kotake S, Nanke Y, Yago T and Yamanaka H: Serum markers of synovitis and bone metabolism in rheumatoid arthritis. Clin Calcium 19: 362-371, 2009 (In Japanese).

26. Chen Q, Jin M, Yang F, Zhu J, Xiao Q and Zhang L: Matrix metalloproteinases: Inflammatory regulators of cell behaviors in vascular formation and remodeling. Mediators Inflamm 2013: 928315, 2013

(c) (i) $\odot$ This work is licensed under a Creative Commons Cy Attribution-NonCommercial-NoDerivatives 4.0 International (CC BY-NC-ND 4.0) License. 